\title{
Endoscopic tumour morphology impacts survival in adenocarcinoma of the oesophagus
}

\author{
Authors \\ William R.C. Knight ${ }^{1}$, Ricardo McEwen ${ }^{1}$, Ben E Byrne ${ }^{1,2}$, Wais Habib ${ }^{1}$, Rebecca Bott ${ }^{1}$, Janine \\ Zylstra $^{1}$, Ula Mahadeva ${ }^{4}$, Oesophageal Cancer Clinical and Molecular Stratification \\ (OCCAMS) Consortium ${ }^{3}$, James A. Gossage ${ }^{1,5,6}$
}

\section{Affiliations:}

1. Department of Surgery, Guy's \& St Thomas' Oesophago-gastric Centre, London, United Kingdom

2. Centre for Surgical Research, Population Health Sciences, Bristol Medical School, University of Bristol, Bristol, UK.

3. Oesophageal Cancer Clinical and Molecular Stratification (OCCAMS) Consortium Cambridge

4. Department of Histopathology, Guy's \& St Thomas', London, United Kingdom

5. Department of Gastroenterology, Guy's \& St Thomas', London, United Kingdom

6. School of Cancer and Pharmaceutical Sciences, King's College London, UK

7. Upper Gastrointestinal Surgery, Department of Molecular Medicine and Surgery, Karolinska Institute, Stockholm, Sweden

\section{Collaborating authors}

Oesophageal Cancer Clinical and Molecular Stratification (OCCAMS) Consortium

Fitzgerald RC, Noorani A, Edwards PAW, Grehan N, Nutzinger B, Hughes C, Fidziukiewicz E, MacRae S, Northrop A, Contino G, Li X, de la Rue R, Katz-Summercorn A, Abbas S, Loureda D, O'Donovan M, Miremadi A, Malhotra S, Tripathi M, Tavaré S, Lynch AG, Eldridge M, Secrier M, Devonshire G, Perner J, Jammula S, Davies J, Crichton C, Carroll N, Safranek P, Hindmarsh A, Sujendran V, Hayes SJ, Ang Y, Sharrocks A, Preston SR, Oakes S, Bagwan I, Save V, Skipworth RJE, Hupp TR, O'Neill JR, Tucker O, Beggs A, Taniere P, Puig S, Underwood TJ, Walker RC, Grace BL, Barr H, Shepherd N, Old O, Lagergren J, Davies A, Chang F, Goh V, Ciccarelli FD, Sanders G, 
Berrisford R, Harden C, Lewis M, Cheong E, Kumar B, Parsons SL, Soomro I, Kaye P, Saunders J, Lovat L, Haidry R, Igali L, Scott M, Sothi S, Suortamo S, Lishman S, Hanna GB, Moorthy K, Peters CJ, Grabowska A, Turkington R, McManus D, Coleman H, Khoo D, Fickling W.

\section{Correspondence:}

William Knight

Department of Surgery, St Thomas' Hospital, London, UK

william.r.knight@gmail.com

Tel +44 (0)7947752228

Key Words: Oesophageal cancer, oesophagectomy, endoscopic tumour morphology, ulcerating tumour, stenosing tumour, stricturing tumour, exophytic tumour. 


\section{Abstract}

Background: Prognostication in oesophageal cancer on the basis of preoperative variables is challenging. Many of the accepted predictors of survival are only derived after surgical treatment and may be influenced by neoadjuvant therapy. This study aims to explore the relationship between pre-treatment endoscopic tumour morphology and postoperative survival.

Methods: Patients with endoscopic descriptions of tumours were identified from the prospectively managed databases including the OCCAMS database. Tumours were classified as exophytic, ulcerating or stenosing. Kaplan Meier survival analysis and multivariable Cox regression analyses were performed to determine hazard ratios (HR) with $95 \%$ confidence intervals.

Results: 262 patients with oesophageal adenocarcinoma undergoing potentially curative resection were pooled from St Thomas' Hospital (161) and the OCCAMS database (101). There were 70 ulcerating, 114 exophytic and 78 stenosing oesophageal adenocarcinomas. Initial tumour staging was similar across all groups (T3/4 tumours 71.4\%, 70.2\%, 74.4\%). Median survival was 55 months, 51 months and 36 months respectively $(p<0.001)$. Rates of lymphovascular invasion $(P=0.0176)$, pathological nodal status $(P=0.0195)$ and pathological T stage $(P=0.0007)$ increased from ulcerating to exophytic to stenosing lesions. Resection margin positivity was $21.4 \%$ in ulcerating tumours compared to $54 \%$ in stenosing tumours $(p<0.001)$. When compared to stenosing lesions, exophytic and ulcerating lesions demonstrated a significant survival advantage on multivariable analysis (HR $0.5695 \% \mathrm{Cl} 0.31-0.93$, HR $0.4295 \% \mathrm{Cl} 0.21-0.82)$.

Conclusion: This study demonstrates that endoscopic morphology may be an important pretreatment prognostic factor in oesophageal cancer. Ulcerating, exophytic and stenosing tumours may represent different pathological processes and tumour biology. 


\section{Introduction}

Oesophageal adenocarcinoma represents a significant oncological and surgical challenge.

Understanding how variables influence survival and patterns of recurrence can help guide multimodality treatment. The problem is that many prognostic variables, such as pathological Tstage and nodal status, tumour regression grade and lympho-vascular invasion, are only available after resection and may themselves be influenced by neoadjuvant therapy. Multi-disciplinary meetings (MDTs) rely on radiological and clinical staging to inform treatment choices yet these variables can be significantly less accurate than the pathological variables which they represent ${ }^{1}$. Therefore, any variable which gives early insight into how a tumour might behave and respond to treatment may prove useful in formulating patient tailored neo-adjuvant treatment strategies.

Endoscopic tumour morphology is a well explored phenomenon in gastric cancer. The Boorman classification describes 4 types of gastric tumour which correspond to differing survival ${ }^{2}$. The Japanese have expanded this system to oesophageal luminal tumours, but mainly squamous cell carcinoma. This study examined three endoscopic tumour morphologies of patients with oesophageal adenocarcinoma; stenosing (stricturing), exophytic (protruding) and ulcerating, to determine if these influence survival. 


\section{Methods}

\section{Study design}

This cohort study was based on a prospectively collected database of consecutive resections performed at a high-volume tertiary oesophago-gastric cancer centre and from the OCCAMS database. Only patients with adenocarcinoma of the gastro-oesophageal junction (Siewert 1,2,3) who had undergone potentially curative oesophagectomy with a pre-treatment endoscopy report describing tumour morphology were included in the study.

\section{Endoscopic morphology}

Endoscopy reports were analysed in retrospect. Patients were categorised into three endoscopic tumour morphologies, ulcerating, exophytic and stenosing. A tumour growing into the lumen was classed as exophytic. This included any tumour described polypoidal. If a tumour was flat with mucosal loss it was deemed ulcerating. If a tumour was impassable it was recorded as stenosing (table 4).

\section{Clinical management}

Patients underwent a standard protocol of investigation including oesophago-gastro-duodenoscopy, computed tomography, endoscopic ultrasound and fluorodeoxyglucose positron emission tomography (FDG-PET). Neo-adjuvant chemotherapy practice evolved during the study period and followed standard indications and regimens as supported by randomised controlled trial evidence ${ }^{3}$. The majority of patients were treated with epirubicn, cisplatin and either $5 \mathrm{FU}$ or capecitabine. Surgical resection included transthoracic or transhiatal oesophagectomy determined by individual surgeon preference. Previous studies in our institution have demonstrated no survival difference between these approaches ${ }^{4}$. Histological staging was standardised to meet the $7^{\text {th }}$ edition TNM criteria, which was the current TNM edition at that time. Adjuvant therapy was determined by the multidisciplinary team consensus based on the positivity of resection margins, presence of lympho- 
vascular invasion, pathological nodal status and the post-operative performance status of the patient.

\section{Statistical analysis}

The following tumour and treatment variables were collected: pre-operative clinical T- and N-stage, Siewert type, neoadjuvant chemotherapy, pathological T- and N-stage, tumour differentiation, Mandard tumour-regression grade (TRG), lympho-vascular invasion (LVI) and circumferential resection margin (CRM). These were compared across morphology groups using chi-squared or Fisher's exact test as appropriate. The primary outcome measure was overall survival.

Overall survival was compared across groups using a Kaplan-Meier plot. Single variable Cox survival regression analysis was performed using pre-treatment variables: cT stage, $\mathrm{CN}$ stage, Siewert type, tumour differentiation, and tumour morphology. Multiple variable regression was conducted with block entry of cT stage, cN stage, and tumour differentiation, with subsequent entry of tumour morphology to determine the incremental improvement in model fit with this additional information. Single and multiple variable regression was similarly performed for post treatment variables: neaoadjuvant chemotherapy, $\mathrm{pT}$ stage, pN stage, LVI, Mandard score, CRM status, as well as tumour differentiation given the known association of this variable with outcome. All analysis was performed using SPSS Statistics version 24 (IBM, Armonk, NY, US). 


\section{Results}

\section{Cohort characteristics}

262 patients were included in the study. 161 patients from a high volume oesophagogastric unit and 101 patients from the OCCAMS database. Patient characteristics are shown in table 1 . There were 70 ulcerating, 114 exophytic and 78 stenosing tumours. Initial clinical T stage of T3/T4 tumours was $71.4 \%$ with ulcerating tumours, $70.2 \%$ with exophytic and $74.4 \%$ with stenosing. In the entire cohort there were 7 cT4 tumours, 2 of which were stenosing on initial endoscopy. Although these tumours were T4 on initial staging, they were later deemed resectable following neo-adjuvant treatment.

$67.1 \%$ of ulcerating tumours and $64.0 \%$ of exophytic tumours had positive nodal disease at clinical staging compared to $76.9 \%$ of patients with stenosing tumours. More patients with stenosing tumours underwent neo-adjuvant chemotherapy (89.7\%) compared to ulcerating $(82.3 \%)$ and exophytic (76\%) tumours. A higher proportion of stenosing tumours had poor or no response to chemotherapy (64.1\%) compared to ulcerating (54.3\%) and exophytic tumours (52.6\%) although this did not reach significance $(P=0.4679) .79 .5 \%$ of stenosing tumours had a $p T 3 / 4$ stage following resection compared to $50 \%$ with ulcerating tumours and $62.3 \%$ of exophytic.

Stenosing tumours were associated with significantly higher rates of lympho-vascular invasion $(71.8 \% \mathrm{p}=0.0176)$ and circumferential resection margin positivity $(56.4 \% \mathrm{p}<0.0001)$ when compared to ulcerating (LVI 51.4\%, CRM 21.4\%) and exophytic tumours (LVI 55.5\%, CRM 29.8\%).

\section{Survival analysis}

Median survival for the cohort overall was 50.3 months. Median survival for stenosing tumours was 36.3 months, 55.8 months for ulcerating tumours and 51.8 months for exophytic tumours $(p=0.0001)$. On Kaplan Meier analysis there were a significant survival difference seen between the three groups ( $p=0.001$ ) (figure 1 ). The association between pre-treatment variables and outcome 
was examined on single and multiple variable analysis (table 2). The addition of tumour morphology significantly improved model fit (chi square change 13.024, $\mathrm{df}=2, p=0.001$ ).

There was a significantly lower risk of death with ulcerating tumours (HR 0.328 95\% Cl 0.172-0.624 p0.001) and exophytic tumours (HR 0.569 95\% I 0.328-0.932 p0.025) compared to stenosing tumours after adjusting for clinical nodal status, clinical T stage and differentiation. Regression analysis was also performed using pathological variables available after surgery. Again, model fit was improved by inclusion of tumour morphology (chi square change $6.116, \mathrm{df}=2, p=0.047$ ). The risk of death was significantly lower with ulcerating (HR $0.5090 .270-0.9600 .037$ ) and exophytic tumours (0.541 0.305$0.962 p=0.036$ ) when compared to stenosing tumour when adjusting for pathological variables (Table 3). 


\section{Discussion}

This study demonstrates that endoscopic tumour morphology is a useful pre-treatment variable which may offer an early insight into tumour biology. Three distinct tumour morphologies have been described here. When adjusted for both pathological and clinical variables, ulcerating and exophytic tumours correspond to an independent survival advantage when compared to stenosing tumours. The sample size was too small to determine if there are genetic determinants to these tumour morphologies.

No study has examined survival of patients with adenocarcinoma according to their initial endoscopic morphology. The Boorman classification system in gastric cancer is well established and the type 1 and 2 show a survival advantage when compared to type 3 and $4^{2}$. The Japanese classification of the macroscopic appearance of non-superficial oesophageal tumours is similar to the Bormann classification. Type 1 is protruding, type 2 is ulcerating, type 3 is ulcerating and infiltrative and type 4 is diffusely infitrative ${ }^{5}$. The Japanese system, however, encompasses both adenocarcinoma and squamous cell carcinoma. Adenocarcinoma of the oesophagus remains a heterogenous disease with complex genetic underpinning and is now considered a separate disease $^{6,7}$. Early histological classification systems of oesophageal adenocarcinoma described medullary (ulcerating), fungating (protruding/exophytic) and scirrhous (scarring/stenosing) ${ }^{8}$. Stenosing tumours have long been known to be associated with a poor prognosis ${ }^{9-13}$. This paper describes a simplified morphological classification system specific to adenocarcinoma of the oesophagus.

The study had several limitations. This was a retrospective study relying on the quality of endoscopy reports where the endoscopists were not classifying tumours according to a prospectively defined classification system. Only patients with clear morphological descriptions of the tumour were included in the study. However, this lack of prospective standardisation may be a source of bias. Image capture was not was not available for all patients and was not used routinely in this study. 
Furthermore, although multivariable adjustments were made using both clinical and pathological variables in this retrospective study, confounders will never be eliminated sufficiently to allow separation between causation and correlation of the involved variables. Although the majority of patients were treated at one institution, the 101 patients from the OCCAMS database came from different institutions making it difficult to fully control for variations in treatments pathways. However, the fact that endoscopic morphology emerged as an independent predictor of survival despite variation across institutions, strengthens the findings of this study. Prospective studies will be needed to validate these findings.

It was thought by the authors on initial analysis that tumour morphologies described here may simply be a reflection of how advanced the tumour was at diagnosis. However, clinical T stage at diagnosis was equivalent in the three groups. $21.8 \%$ of stenosing tumours were clinically staged at T1-2. Even if the stenosing tumours are a result of a more advanced disease, this was not discernible on imaging, confirming the importance of the morphology characteristics.

In this analysis, stenosing tumours are associated with higher rates of adverse prognostic factors; lympho-vascular invasion, poor differentiation, pathological nodal status, poor response to chemotherapy and higher resection margin positivity. These patients are also likely to suffer from poor nutrition and weight loss, which is associated with worse outcomes ${ }^{14}$.

Studies looking at gastric cancer have shown Bormann type 1 (exophytic) and 2 (ulcerating) share similar survival curves, with types 3 and 4 showing progressively worse outcomes ${ }^{2}$. In this cohort there appeared to be a survival advantage with ulcerating tumours when compared to exophytic tumours, although this did not reach significance on multivariable analysis. This was not an expected finding. It was theorised that exophytic tumours, growing luminally, would localise the tumour whilst ulcerating tumours, growing towards the adventitia, would show higher rates of LVI and therefore a worse prognosis. Studies have shown that superficial ulcerating (type 0-iii) lesions in the stomach and oesophagus differ ${ }^{15}$. In the stomach these ulcerating lesions contain viable tumour at the more 
superficial periphery of the ulcer and do not permeate into the submucosa. Barrett's associated Type 0-iii lesions in the oesophagus show viable tumour at the base of the ulcer. It would follow that if the precursor ulcerating lesions of the oesophagus have tumour invading the submucosa then this would translate to a survival disadvantage. This does not appear to be the case. Siewert has postulated that the overall survival advantage seen with $\mathrm{AC}$ compared to squamous cell carcinoma may be due to inflammation associated with the Barrett's pathophysiology ${ }^{16}$. This inflammation is said to have a disrupting effect on the lymphovasculature which has an initially protective effect on tumour dissemination. It is possible that disruption of the lymphovasculature may also explain the relative survival advantage seen with ulcerating tumours. However, further studies will be needed to determine this.

In conclusion this study has examined the survival outcomes of three distinct endoscopic morphologies, ulcerating, exophytic and stenosing tumours. Ulcerating and exophytic tumours are associated with independent survival advantage compared to stenosing tumours. Further prospective studies will be needed to validate these findings. 


\section{Tables and figures}

Table 1

\begin{tabular}{|c|c|c|c|c|c|c|c|c|c|}
\hline \multirow{3}{*}{$\begin{array}{l}\text { Variable } \\
\text { Number }\end{array}$} & \multicolumn{2}{|c|}{ Cohort } & \multicolumn{2}{|c|}{ Ulcerating } & \multicolumn{2}{|c|}{ Exophytic } & \multicolumn{2}{|c|}{ Stenosing } & \multirow[b]{2}{*}{$P$} \\
\hline & $\mathrm{N}$ & $\%$ & $\mathrm{~N}$ & $\%$ & $\mathrm{~N}$ & $\%$ & $\mathrm{~N}$ & $\%$ & \\
\hline & \multicolumn{2}{|l|}{262} & 70 & & 114 & & 78 & & \\
\hline \multicolumn{10}{|l|}{ cT } \\
\hline T1-2 & 65 & $24.8 \%$ & 17 & $24.3 \%$ & 31 & $27.2 \%$ & 17 & $21.8 \%$ & $P=0.7212$ \\
\hline T3/4 & 188 & $71.8 \%$ & 50 & $71.4 \%$ & 80 & $70.2 \%$ & 58 & $74.4 \%$ & \\
\hline \multicolumn{10}{|l|}{$\mathrm{cN}$} \\
\hline cNO & 78 & $29.8 \%$ & 23 & $32.9 \%$ & 38 & $33.0 \%$ & 17 & $21.8 \%$ & $P=0.1739$ \\
\hline cN1,2,3 & 180 & $68.7 \%$ & 47 & $67.1 \%$ & 73 & $64.0 \%$ & 60 & $76.9 \%$ & \\
\hline \multicolumn{10}{|l|}{ Tumour location } \\
\hline Siewert type 1 & 113 & $43.1 \%$ & 32 & $45.7 \%$ & 49 & $43.0 \%$ & 32 & $41.0 \%$ & $P=0.2916$ \\
\hline Siewert type 2 & 113 & $43.1 \%$ & 24 & $34.3 \%$ & 51 & $44.7 \%$ & 38 & $48.7 \%$ & \\
\hline Siewert type 3 & 24 & $9.2 \%$ & 10 & $14.3 \%$ & 9 & $7.9 \%$ & 5 & $6.4 \%$ & \\
\hline \multicolumn{10}{|l|}{ Neo-adjuvant treatment } \\
\hline NAC & 215 & $82.1 \%$ & 58 & $82.3 \%$ & 87 & $76 \%$ & 70 & $89.7 \%$ & $P=0.057$ \\
\hline Surgery alone & 47 & $17.9 \%$ & 12 & $17.1 \%$ & 27 & $24 \%$ & 8 & $13.2 \%$ & \\
\hline \multicolumn{10}{|l|}{ pT } \\
\hline CPR & 17 & $6.5 \%$ & 7 & $10.0 \%$ & 4 & $3.5 \%$ & 4 & $5.1 \%$ & $P=0.0007$ \\
\hline pT1/2 & 77 & $29.4 \%$ & 28 & $40.0 \%$ & 38 & $33.3 \%$ & 11 & $14.1 \%$ & \\
\hline pT3/4 & 168 & $64.1 \%$ & 35 & $50.0 \%$ & 71 & $62.3 \%$ & 62 & $79.5 \%$ & \\
\hline \multicolumn{10}{|l|}{ pN } \\
\hline pNO & 106 & $40.1 \%$ & 35 & $50.0 \%$ & 49 & $43.0 \%$ & 22 & $28 \%$ & $P=0.0195$ \\
\hline pN1 & 45 & $17.2 \%$ & 10 & $14.2 \%$ & 22 & $19.3 \%$ & 13 & $16.7 \%$ & \\
\hline $\mathrm{pN} 2 / 3$ & 108 & $41.2 \%$ & 24 & $34.3 \%$ & 42 & $36.8 \%$ & 42 & $53.8 \%$ & \\
\hline \multicolumn{10}{|l|}{ Pathological grade } \\
\hline Poorly differentiated & 138 & $52.7 \%$ & 35 & $50.0 \%$ & 53 & $46.5 \%$ & 50 & $64.1 \%$ & $P=0.0797$ \\
\hline Mod differentiated & 109 & $41.6 \%$ & 28 & $40.0 \%$ & 55 & $48.2 \%$ & 26 & $33.3 \%$ & \\
\hline \multicolumn{10}{|l|}{ Mandard score } \\
\hline 1 Complete response & 17 & $6.5 \%$ & 7 & $10.0 \%$ & 4 & $3.5 \%$ & 4 & $5.1 \%$ & $P=0.4679$ \\
\hline 2-3 Partial or Good & 44 & $16.8 \%$ & 10 & $14.2 \%$ & 18 & $15.8 \%$ & 16 & $20.5 \%$ & \\
\hline 4-5 Poor or No response & 148 & $56.5 \%$ & 38 & $54.3 \%$ & 60 & $52.6 \%$ & 50 & $64.1 \%$ & \\
\hline \multicolumn{10}{|l|}{ LVI } \\
\hline Yes & 153 & $58.4 \%$ & 36 & $51.4 \%$ & 61 & $53.5 \%$ & 56 & $71.8 \%$ & $P=0.0176$ \\
\hline No & 104 & $39.7 \%$ & 33 & $47.1 \%$ & 50 & $43.9 \%$ & 21 & $26.9 \%$ & \\
\hline \multicolumn{10}{|l|}{ CRM } \\
\hline RO & 163 & $62.2 \%$ & 53 & $75.7 \%$ & 76 & $66.7 \%$ & 34 & $43.6 \%$ & $P<0.0001$ \\
\hline R1 & 93 & $35.5 \%$ & 15 & $21.4 \%$ & 34 & $29.8 \%$ & 44 & $56.4 \%$ & \\
\hline \multicolumn{10}{|l|}{ Median survival } \\
\hline Months & & 50.3 & & 55.8 & & 51.8 & & 36.3 & $P=0.0001$ \\
\hline
\end{tabular}


Table 2 Univariable and multivariable analysis with pre-treatment variables

\begin{tabular}{|c|c|c|c|c|c|c|c|c|c|c|c|c|}
\hline \multirow[t]{2}{*}{ Characteristic } & \multicolumn{4}{|c|}{ Single variable analysis } & \multicolumn{4}{|c|}{$\begin{array}{l}\text { Multiple variable without } \\
\text { morphology }\end{array}$} & \multicolumn{4}{|c|}{$\begin{array}{l}\text { Multiple variable with } \\
\text { morphology }\end{array}$} \\
\hline & $\mathrm{HR}$ & $\begin{array}{l}95 \% \\
\mathrm{Cl}\end{array}$ & & $p$ & $\mathrm{HR}$ & $\begin{array}{l}95 \% \\
\mathrm{Cl}\end{array}$ & & $p$ & $\mathrm{HR}$ & $\begin{array}{l}95 \% \\
\mathrm{Cl}\end{array}$ & & $p$ \\
\hline & & Lower & Upper & & & Lower & Upper & & & Lower & Upper & \\
\hline cT1/2 & Ref & & & & Ref & & & & Ref & & & \\
\hline cT3/4 & 1.815 & 1.082 & 3.046 & .024 & 1.704 & 0.920 & 3.157 & .090 & 1.735 & 0.953 & 3.157 & .071 \\
\hline cNO & Ref & & & & Ref & & & & Ref & & & \\
\hline $\mathrm{cN} 1 / 2 / 3$ & 1.220 & 0.774 & 1.924 & .392 & 0.820 & 0.473 & 1.422 & .480 & 0.825 & 0.484 & 1.406 & .480 \\
\hline Siewert 1 & Ref & & & & Ref & & & & Ref & & & \\
\hline Siewert 2 & 0.928 & 0.599 & 1.437 & .737 & 0.817 & 0.514 & 1.299 & .392 & 0.707 & 0.443 & 1.130 & .147 \\
\hline Siewert 3 & 0.697 & 0.314 & 1.551 & .377 & 0.516 & 0.228 & 1.168 & .112 & 0.593 & 0.262 & 1.344 & .211 \\
\hline Well/mod diff & Ref & & & & Ref & & & & Ref & & & \\
\hline Poor diff & 1.977 & 1.249 & 3.128 & .004 & 2.129 & 1.317 & 3.441 & .002 & 1.994 & 1.219 & 3.262 & .006 \\
\hline Stenosing & Ref & & & & - & & & & Ref & & & \\
\hline Exophytic & 0.532 & 0.338 & 0.838 & .007 & & & & & 0.569 & 0.347 & 0.932 & .025 \\
\hline Ulcerated & 0.332 & 0.184 & 0.598 & $<.001$ & & & & & 0.328 & 0.172 & 0.624 & .001 \\
\hline
\end{tabular}

Table 3Univariable and multivariable analysis with post treatment variables

\begin{tabular}{|c|c|c|c|c|c|c|c|c|c|c|c|c|}
\hline \multirow[t]{2}{*}{ Characteristic } & \multicolumn{4}{|c|}{ Single variable analysis } & \multicolumn{4}{|c|}{$\begin{array}{l}\text { Multiple variable without } \\
\text { morphology }\end{array}$} & \multicolumn{4}{|c|}{$\begin{array}{l}\text { Multiple variable with } \\
\text { morphology }\end{array}$} \\
\hline & $\mathrm{HR}$ & $\begin{array}{l}95 \% \\
\mathrm{Cl}\end{array}$ & & $p$ & $\mathrm{HR}$ & $\begin{array}{l}95 \% \\
\mathrm{Cl}\end{array}$ & & $p$ & $\mathrm{HR}$ & $\begin{array}{l}95 \% \\
\mathrm{Cl}\end{array}$ & & $p$ \\
\hline & & Lower & Upper & & & Lower & Upper & & & Lower & Upper & \\
\hline NAC & Ref & & & & Ref & & & & Ref & & & \\
\hline No chemo & 0.597 & 0.325 & 1.096 & .096 & 0.000 & 0.000 & $2 \mathrm{E} 227$ & .970 & 0.000 & 0.000 & $5 \mathrm{E} 219$ & .969 \\
\hline pCR & Ref & & & & Ref & & & & Ref & & & \\
\hline pT1/2 & 1.515 & 0.339 & 6.773 & .587 & 1.086 & 0.121 & 9.703 & .941 & 1.143 & 0.128 & 10.236 & .095 \\
\hline pT3/4 & 6.381 & 1.564 & 26.036 & .010 & 1.451 & 0.171 & 12.319 & .733 & 1.525 & 0.180 & 12.929 & .699 \\
\hline pNO & Ref & & & & Ref & & & & Ref & & & \\
\hline pN1 & 3.085 & 1.584 & 6.010 & .001 & 2.051 & .891 & 4.720 & .091 & 2.162 & 0.939 & 4.979 & .070 \\
\hline $\mathrm{pN} 2 / 3$ & 5.747 & 3.287 & 10.049 & $<.001$ & 2.358 & 1.051 & 5.290 & .037 & 2.394 & 1.059 & 5.410 & .036 \\
\hline $\begin{array}{l}\text { Well/mod } \\
\text { diff }\end{array}$ & Ref & & & & Ref & & & & Ref & & & \\
\hline Poor diff & 1.977 & 1.249 & 3.128 & .004 & 0.990 & 0.576 & 1.700 & .971 & 0.910 & .526 & 1.573 & .734 \\
\hline LVI negative & Ref & & & & Ref & & & & Ref & & & \\
\hline LVI positive & 4.639 & 2.759 & 7.800 & $<.001$ & 2.533 & 1.210 & 5.303 & .014 & 2.519 & 1.188 & 5.340 & .016 \\
\hline TRG 1 & Ref & & & & $*$ & & & & $*$ & & & \\
\hline TRG 2/3 & 2.504 & 0.548 & 11.441 & .236 & Ref & & & & Ref & & & \\
\hline TRG 3/4 & 5.557 & 1.355 & 22.782 & .017 & 1.345 & 0.661 & 2.735 & .413 & 1.313 & 0.638 & 2.835 & .460 \\
\hline CRM -ve & Ref & & & & Ref & & & & Ref & & & \\
\hline CRM +ve & 3.545 & 2.336 & 5.381 & $<.001$ & 1.926 & 1.138 & 3.260 & .015 & 1.637 & 0.946 & 2.835 & .078 \\
\hline Stenosing & Ref & & & & - & & & & Ref & & & \\
\hline Exophytic & 0.532 & 0.338 & 0.838 & .007 & & & & & 0.541 & 0.305 & 0.962 & .036 \\
\hline Ulcerated & 0.332 & 0.184 & 0.598 & $<.001$ & & & & & 0.509 & 0.270 & 0.960 & .037 \\
\hline
\end{tabular}




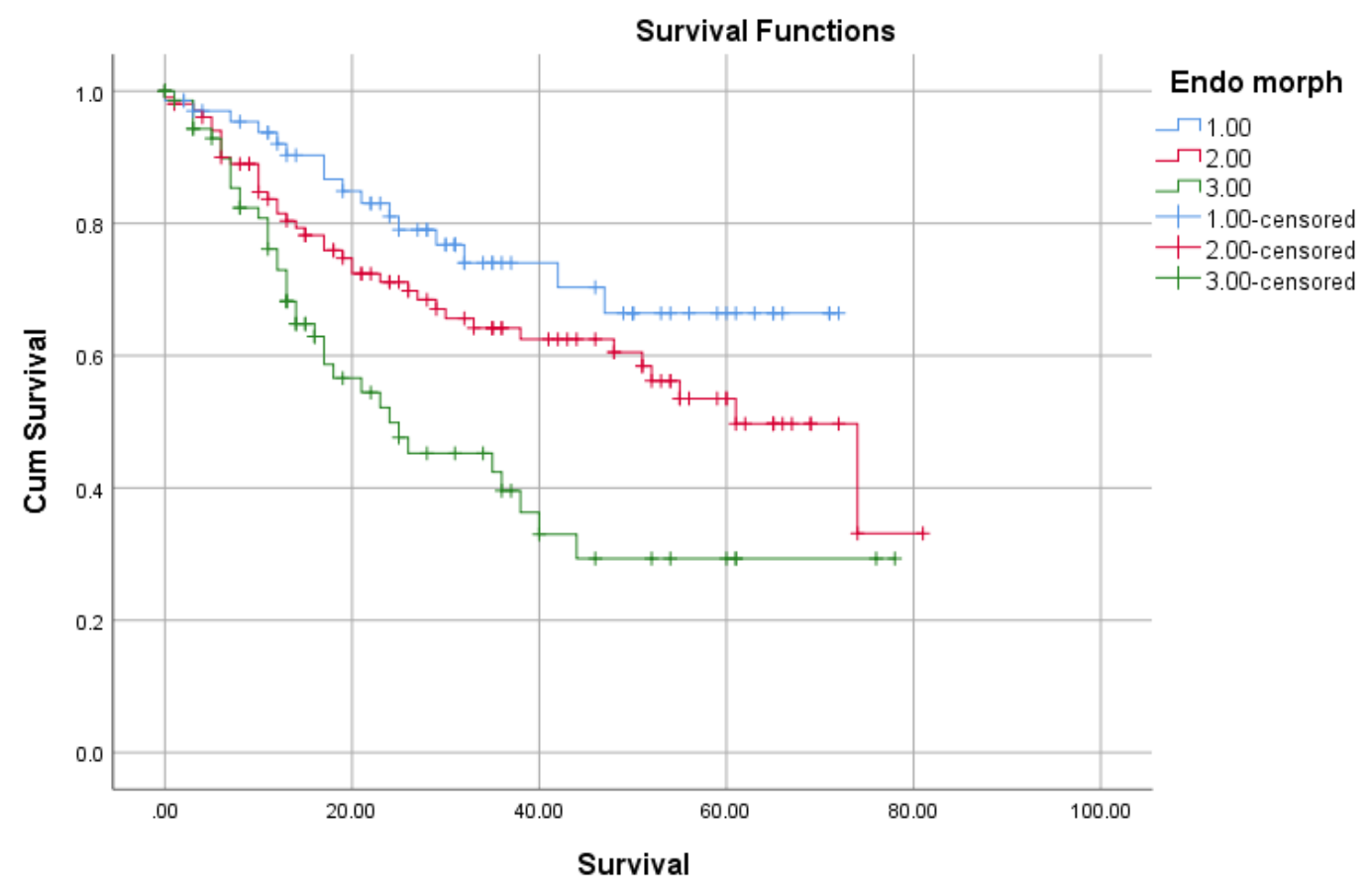

Overall Comparisons

Chi-Square df $\quad$ Sig.

\begin{tabular}{ll|l|l}
\hline Log Rank (Mantel-Cox) & 16.535 & 2 & .000 \\
\hline
\end{tabular}

Test of equality of survival distributions for the different levels of

Endo morph.

Table 4 Tumour endoscopic morphology classification

\begin{tabular}{|l|l|}
\hline Tumour type & Description \\
\hline Ulcerating & $\begin{array}{l}\text { Any tumour where there is marked ulceration and mucosal loss. } \\
\text { May have intraluminal components especially at the edge of the tumour but overall } \\
\text { tumour extends towards the adventitia }\end{array}$ \\
\hline Exophytic & $\begin{array}{l}\text { Where the majority of the tumour extends intraluminally as seen endoscopically } \\
\text { There may be a polypoidal component }\end{array}$ \\
\hline Stenosing & Where the lumen is narrowed and the scope is impassable \\
\hline
\end{tabular}


1. Yip C, Cook GJR, Landau DB, Davies A, Goh V. Performance of different imaging modalities in assessment of response to neoadjuvant therapy in primary esophageal cancer. Dis Esophagus. 2016;29(2):116-130. doi:10.1111/dote.12315

2. Li C, Oh SJ, Kim S, et al. Macroscopic borrmann type as a simple prognostic indicator in patients with advanced gastric cancer. Oncology. 2009;77(3-4):197-204. doi:10.1159/000236018

3. Cunningham D, Allum WH, Stenning SP, et al. Perioperative Chemotherapy versus Surgery Alone for Resectable Gastroesophageal Cancer. N Engl J Med. 2006;355(1):11-20. doi:10.1056/NEJMoa055531

4. Davies AR, Sandhu H, Pillai A, et al. Surgical resection strategy and the influence of radicality on outcomes in oesophageal cancer. Br J Surg. 2014;101(5):511-517. doi:10.1002/bjs.9456

5. Japanese Classification of Esophageal Cancer, 11th Edition: part I. Esophagus. 2017;14(1):136. doi:10.1007/s10388-016-0551-7

6. Bornschein J, Wernisch L, Secrier M, et al. Transcriptomic profiling reveals three molecular phenotypes of adenocarcinoma at the gastroesophageal junction. Int J Cancer. 2019;145(12):3389-3401. doi:10.1002/ijc.32384

7. Rü Diger Siewert J, Ott K. Are Squamous and Adenocarcinomas of the Esophagus the Same Disease? Semin Radiat Oncol. 17:38-44. doi:10.1016/j.semradonc.2006.09.007

8. Liu FS, Zhou CN. Pathology of Carcinoma of the Esophagus. In: Carcinoma of the Esophagus and Gastric Cardia. Springer Berlin Heidelberg; 1984:77-116. doi:10.1007/978-3-642-69183$6 \_4$

9. Deng H-Y, Alai G, Luo J, Li G, Zhuo Z-G, Lin Y-D. Cancerous esophageal stenosis before treatment was significantly correlated to poor prognosis of patients with esophageal cancer: a meta-analysis. J Thorac Dis. 2018;10(7):4212-4219. doi:10.21037/jtd.2018.06.89

10. Sillah K, Pritchard SA, Watkins GR, et al. The degree of circumferential tumour involvement as a prognostic factor in oesophageal cancer. Eur J Cardio-thoracic Surg. 2009;36(2):368-373 doi:10.1016/j.ejcts.2008.12.052

11. Bhutani MS, Barde CJ, Markert RJ, Gopalswamy N. Length of esophageal cancer and degree of luminal stenosis during upper endoscopy predict $\mathrm{T}$ stage by endoscopic ultrasound. Endoscopy. 2002;34(6):461-463. doi:10.1055/s-2002-31996

12. Yang YS, Hu WP, Ni PZ, Wang WP, Yuan Y, Chen LQ. Esophageal luminal stenosis is an independent prognostic factor in esophageal squamous cell carcinoma. Oncotarget. 2017;8(26):43397-43405. doi:10.18632/oncotarget.14762

13. Deng H-Y, Li G, Luo J. Does oesophageal stenosis have any impact on survival of oesophageal cancer patients? Interact Cardiovasc Thorac Surg. 2018;27(3):384-386. doi:10.1093/icvts/ivy095

14. Hikage M, Taniyama Y, Sakurai T, et al. The Influence of the Perioperative Nutritional Status on the Survival Outcomes for Esophageal Cancer Patients with Neoadjuvant Chemotherapy. Ann Surg Oncol. December 2019. doi:10.1245/s10434-019-07742-9

15. Axon A, Diebold MD, Fujino M, et al. Update on the Paris classification of superficial neoplastic lesions in the digestive tract. Endoscopy. 2005;37(6):570-578. doi:10.1055/s-2005861352

16. Siewert JR, Ott K. Are squamous and adenocarcinomas of the esophagus the same disease? 
Semin Radiat Oncol. 2007;17(1):38-44. doi:10.1016/j.semradonc.2006.09.007 